\title{
Evolution of the coupling constant in SU(2) lattice gauge theory with two adjoint fermions
}

\author{
Ari J. Hietanen \\ Department of Physics, Florida International University \\ Kari Rummukainen \\ Department of Physics and Helsinki Institute of Physics, University of Helsinki, Finland \\ Kimmo TuOMinen \\ Department of Physics, University of Jyväskylä and Helsinki Institute of Physics, University of Helsinki, Finland
}

\begin{abstract}
We measure the evolution of the coupling constant using the Schrödinger functional method in the lattice formulation of $\mathrm{SU}(2)$ gauge theory with two massless Dirac fermions in the adjoint representation. We observe strong evidence for an infrared fixed point, where the theory becomes conformal. We measure the $\beta$-function and the coupling constant as a function of the energy scale.
\end{abstract}

PACS numbers: 11.15.Ha,12.60.Nz

\section{INTRODUCTION}

In preparation for the phenomenology at LHC, there has recently been increased interest in field theories which appear (quasi) conformal when probed towards infrared, i.e. over large distance scales. There are basically two novel applications which utilize such theories, namely walking technicolor [1] and unparticles [2, 3]. For model building along these directions it is very desirable to single out candidate theories which allow for the existence of a nontrivial infrared fixed point and then use these as a basis for more detailed analyses. To determine the location of the conformal window as a function of the number of colors, flavors and fermion representations is a nonperturbative problem. Traditional way to obtain semiquantitative estimates is the so-called rainbow approximation to the Schwinger-Dyson equation for the nonperturbative fermion propagator [4, 5, 6]. More recent developments include analytic $\beta$-function ansätze [7, 8, 9] and analyses on the role of topological excitations [10]. Phenomenological constraints on walking technicolor require that conformal behavior is obtained with only modest amount of new matter fields, and utilizing higher representation fermions, several plausible candidates have been proposed in [11, 12]. Although subject to uncertainties, these methods are invaluable in providing hints towards theories which are of phenomenological interest and motivate their analyses using lattice methods.

Guided by the phase diagrams of [1], initial lattice studies of two and three color theories with two fermion flavours in the two-index symmetric representation have recently appeared [13, 14, 15, 16, 17, 18]. For related studies with fundamental fermions in $\mathrm{SU}(3)$ gauge theory, see [19, 20, 21, 22, 23].

In this work we study the evolution of the coupling constant of the candidate model with "minimal" field content, consisting of the standard $\mathrm{SU}(2)$ gauge field and two massless Dirac fermions in the adjoint representa- tion. The euclidean Lagrange density is

$$
\mathcal{L}=\frac{1}{2} \operatorname{Tr} F_{\mu \nu} F_{\mu \nu}+\sum_{f} \bar{\psi}_{f} i \gamma_{\mu} D_{\mu} \psi_{f}
$$

with $D_{\mu}=\partial_{\mu}+i g A^{a} T^{a}$, where $\left(T^{a}\right)^{b c}=-i \epsilon^{a b c}$ are the generators of the adjoint representation. To obtain the evolution of the couplig we use the lattice Schrödinger functional scheme, which has been very successfully applied to e.g. two-flavour QCD by the Alpha collaboration 24].

Perturbatively, the two-loop $\beta$-function of this theory with $N_{f}$ adjoint fermions is

$$
\beta(g)=\mu \frac{\mathrm{d} g}{\mathrm{~d} \mu}=-\beta_{0} \frac{g^{3}}{16 \pi^{2}}-\beta_{1} \frac{g^{5}}{\left(16 \pi^{2}\right)^{2}},
$$

where $\beta_{0}=\left(22-8 N_{f}\right) / 3$ and $\beta_{1}=4\left(34-32 N_{f}\right) / 3$. For $N_{f}=1,2$ the coefficient $\beta_{0}$ is positive while $\beta_{1}$ is positive for $N_{f}=1$ but negative for $N_{f}=2$. Hence, for $N_{f}=2$ there is a zero of the two-loop $\beta$-function at $g_{*}^{2}=-\left(\beta_{0} / \beta_{1}\right) 16 \pi^{2} \approx 7.9$, i.e. $\alpha_{*}=g_{*}^{2} /(4 \pi) \approx 0.6$, corresponding to an infrared fixed point (IRFP). Large value of $g_{*}^{2}$ casts doubt on the validity of the perturbative result and nonperturbative measurement of the running coupling on the lattice is required. Indeed, as we report below, the lattice simulations reveal large deviations from the two-loop perturbative behaviour.

We note that the $\beta$-function has been calculated up to four loops in the Minimal Subtraction (MS) scheme [25]. Beyond two loops the $\beta$-function is scheme dependent and the result cannot be directly compared with the lattice Schrödinger functional scheme used here.

For the gauge group $\mathrm{SU}(2)$ considered here the twoindex symmetric representation coincides with the adjoint representation. We have recently performed largevolume high-statistics lattice simulations of this theory, concentrating on the chiral properties of the mass spectrum [26, 27]. The main result of this study was that at small enough lattice gauge coupling, in contrast to 
QCD, we did not observe spontaneous chiral symmetry breaking as the quark mass was decreased. Because the chiral condensate generates a mass scale, its existence would exclude the IRFP. While the non-observation of the spontaneous chiral symmetry breaking is very suggestive, it by no means proves that the IRFP exists at non-zero coupling, and one needs to measure the evolution of the coupling directly ${ }^{1}$.

\section{LATTICE FORMULATION}

The lattice theory is defined with the action

$$
S=S_{\mathrm{G}}+S_{\mathrm{F}},
$$

where $S_{\mathrm{G}}$ is the standard Wilson plaquette action for $\mathrm{SU}(2)$ gauge fields:

$$
S_{\mathrm{G}}=\beta_{L} \sum_{x, \mu<\nu}\left(1-\frac{1}{2} \operatorname{Tr}\left[U_{x, \mu} U_{x+\hat{\mu}, \nu} U_{x+\hat{\nu}, \mu}^{\dagger} U_{x, \nu}^{\dagger}\right]\right) .
$$

$S_{\mathrm{F}}$ is the Wilson fermion action for two Dirac fermions in the adjoint representation of $\mathrm{SU}(2)$ :

$$
S_{\mathrm{F}}=\sum_{f=\mathrm{u}, \mathrm{d}} \sum_{x, y} \bar{\psi}_{f, x} M_{x y} \psi_{f, y},
$$

where

$$
M_{x y}=\delta_{x y}-\kappa \sum_{\mu}\left[\left(1+\gamma_{\mu}\right) V_{x, \mu}+\left(1-\gamma_{\mu}\right) V_{x-\mu, \mu}^{T}\right] .
$$

It differs from the standard Wilson fermion action only by the replacement of the fundamental representation link matrices $U_{\mu}(x)$ with the adjoint representation ones:

$$
V_{\mu}^{a b}(x)=2 \operatorname{Tr}\left(\lambda^{a} U_{\mu}(x) \lambda^{b} U_{\mu}^{\dagger}(x)\right),
$$

where $\lambda^{a}=\frac{1}{2} \sigma^{a}, a=1,2,3$, are the generators of the fundamental representation. The lattice action is conventionally parametrised with two dimensionless parameters,

$$
\beta_{L}=\frac{4}{g_{0}^{2}}, \quad \kappa=\frac{1}{8+2 a m_{q, 0}},
$$

where $a$ is the lattice spacing, and $g_{0}^{2}$ and $m_{q, 0}$ the bare gauge coupling and quark mass. Because the Wilson action explicitly breaks the chiral symmetry of the continuum quark action, quark masses are additively renormalised and the bare quark mass must be tuned to achieve the massless limit. In order to keep the methodology simple, we do not implement $O(a)$ improvement on the lattice.

\footnotetext{
1 Alternatively one can study the properties of the physical degrees of freedom in the limit of zero quark mass as outlined in [28].
}

In earlier studies [13, 26] it has been found that in this lattice theory there exists a critical coupling $\beta_{L, c} \approx 2$ so that when $\beta_{L}<\beta_{L, c}$ the theory shows signs of spontaneous chiral symmetry breaking, but reaching the limit $m_{Q} \rightarrow 0$ is prevented by the appearance of a first order transition where $m_{Q}$ jumps to negative values. However, when $\beta_{L}>\beta_{L, c}$ the $m_{Q} \rightarrow 0$ limit can be reached without observing the spontaneous chiral symmetry breaking. This is the region where it is possible to find conformal behaviour in the limit of massless fermions.

Because the Wilson fermion action breaks the continuum chiral symmetry, the quark mass is additively renormalised and we determine the quark mass through the $\mathrm{PCAC}$ relation

$$
2 m_{Q}=\left[\partial_{t}\left\langle V_{A}(x)\right\rangle /\left\langle V_{P}(x)\right\rangle\right]_{\left.\right|_{x_{0}=L / 2}}
$$

where $V_{A}$ and $V_{P}$ are (non-improved) axial and pseudoscalar current correlators [29]:

$$
\begin{aligned}
& V_{A}(x)=a^{6} \sum_{\boldsymbol{y}, \boldsymbol{z}}\left\langle\overline{\mathrm{d}}(x) \gamma_{0} \gamma_{5} \mathrm{u}(x) \overline{\mathrm{u}}(\boldsymbol{y}, 0) \gamma_{5} \mathrm{~d}(\boldsymbol{z}, 0)\right\rangle \\
& V_{P}(x)=a^{6} \sum_{\boldsymbol{y}, \boldsymbol{z}}\left\langle\overline{\mathrm{d}}(x) \gamma_{5} \mathrm{u}(x) \overline{\mathrm{u}}(\boldsymbol{y}, 0) \gamma_{5} \mathrm{~d}(\boldsymbol{z}, 0)\right\rangle
\end{aligned}
$$

Here $\mathrm{d}$ and $\mathrm{u}$ refer to two quark flavours, and at time slices $x_{0}=0$ and $x_{0}=L$ the gauge fields are fixed to specific Schrödinger function boundary values described in detail below. For each lattice coupling $\beta_{L}$ we tune $\kappa$ so that $m_{Q}$ vanishes. This determines the critical line $\kappa_{c}\left(\beta_{L}\right)$; from now on we assume that we always tune to the limit $m_{Q}=0$.

The coupling is measured using the Schrödinger functional (SF) method [30, 31, 32], i.e. introducing a background field using special boundary conditions and studying the response of the system to changes of the background field. The scale at which the coupling is measured is determined by the finite size of the system. Here we consider lattices of volume $V=L^{4}=(N a)^{4}$, and following [33], the spatial gauge links on the $x_{0}=0$ and $x_{0}=L$ boundaries are fixed to constant diagonal $\mathrm{SU}(2)$ matrices:

$$
\begin{aligned}
U_{\mu}\left(x_{0}=0\right) & =\exp \left(-i \eta \sigma_{3} a / L\right) \\
U_{\mu}\left(x_{0}=L\right) & =\exp \left(-i(\pi-\eta) \sigma_{3} a / L\right),
\end{aligned}
$$

where $\sigma_{3}$ is the third Pauli matrix. The spatial gauge field boundary conditions are periodic. During the simulation the fermion fields are set to vanish at boundaries $x_{0}=0$ and $x_{0}=L$, and are "twisted" periodic to spatial directions: $\psi\left(x+L \hat{e}_{i}\right)=\exp (i \pi / 5) \psi(x)$ [32].

At the classical level the gauge field boundary conditions generate a constant color diagonal chromoelectric field, and the derivative of the action with respect to $\eta$ is easily calculable:

$$
\frac{\partial S^{\mathrm{cl}} .}{\partial \eta}=\frac{k}{g_{0}^{2}}
$$




\begin{tabular}{|l|l|l|}
\hline$\beta_{L}$ & $\kappa_{c}$ & $m_{Q} a$ \\
\hline 2.05 & 0.18625 & $-0.00377(29)$ \\
2.2 & 0.1805 & $0.00016(16)$ \\
2.5 & 0.17172 & $-0.00079(10)$ \\
3 & 0.161636 & $0.00213(11)$ \\
3.5 & 0.155132 & $0.00028(11)$ \\
4.5 & 0.14712 & $0.00000(05)$ \\
8 & 0.136415 & $-0.00038(04)$ \\
\hline
\end{tabular}

TABLE I: Values of $\beta_{L}$ and the estimates of $\kappa_{c}$ used. $\kappa_{c}$ is determined on $16^{4}$ lattices with small statistics runs. The table also shows the small residual value of $m_{Q} a$ measured from the much larger statistics production runs on $16^{4}$ lattices.

where, on a lattice of size $L^{4}=(N a)^{4}[33]$,

$$
k=-24(L / a)^{2} \sin \frac{\pi-2 \eta}{(L / a)^{2}} .
$$

At quantum level the boundary conditions generate a background field. Using an effective action $\Gamma$,

$$
e^{-\Gamma}=\int \mathcal{D}[U, \psi, \bar{\psi}] e^{-S},
$$

the coupling constant is defined through

$$
\frac{\partial \Gamma}{\partial \eta}=\left\langle\frac{\partial S_{\mathrm{G}}}{\partial \eta}\right\rangle=\frac{k}{g^{2}(L)} .
$$

We fix $\eta=\pi / 4$ after taking the derivative. For our action the observable $\left\langle\partial S_{\mathrm{G}} / \partial \eta\right\rangle$ is proportional to the expectation value of the boundary plaquette and easily measurable. The result is the coupling constant $g^{2}(L)$ as a function of the physical size of the lattice. Because a priori the lattice spacing $a$ is unknown, we denote the lattice measurements with $g^{2}\left(L / a, \beta_{L}\right)$. Formal continuum limit is obtained by keeping $g^{2}$ constant while taking $L / a \rightarrow \infty$.

\section{RESULTS AND ANALYSIS}

As stated in the previous section we will consider lattice couplings $\beta_{L}<\beta_{L, c} \approx 2$ and tune to the $m_{Q}=0$ limit corresponding to a critical value $\kappa_{c}\left(\beta_{L}\right)$. The values of $\kappa_{c}$, as well as the corresponding $m_{Q}$, at each value of $\beta_{L}$ used in this study are shown in Table प. The $\beta_{L^{-}}$ values which we consider are all larger than the critical value $\beta_{L, c} \approx 2$; indeed, already at $\beta_{L}=1.9$ it was impossible to reach $m_{Q}=0$ limit.

The measurements of $g^{2}\left(L / a, \beta_{L}\right)$ are done on lattices with volumes $V / a^{4}=4^{4}-20^{4}$, with the results shown in Fig. 1 and tabulated in Table III] We use a hybrid Monte Carlo (HMC) algorithm, with trajectory length $\approx 1$; the table also shows the number of HMC trajectories for each point. The measurement of the coupling is done at the end of each trajectory. The acceptance rate in the

\begin{tabular}{|l|r|r|l|}
\hline$\beta_{L}$ & $N$ & $N_{\text {traj. }}$ & $1 / g^{2}$ \\
\hline 2.05 & 4 & 20000 & $0.2504(17)$ \\
& 6 & 51200 & $0.2233(16)$ \\
& 8 & 29000 & $0.2179(36)$ \\
& 12 & 27000 & $0.2187(33)$ \\
& 16 & 30900 & $0.2391(53)$ \\
& 20 & 17300 & $0.257(22)$ \\
\hline 2.2 & 4 & 16000 & $0.3069(17)$ \\
& 6 & 51200 & $0.2857(15)$ \\
& 8 & 19900 & $0.2719(36)$ \\
& 12 & 55000 & $0.2764(30)$ \\
& 16 & 30141 & $0.2857(54)$ \\
& 20 & 8000 & $0.284(17)$ \\
\hline 2.5 & 4 & 15000 & $0.3990(16)$ \\
& 6 & 51200 & $0.3815(14)$ \\
& 8 & 21500 & $0.3653(32)$ \\
& 12 & 37400 & $0.3621(39)$ \\
& 16 & 33000 & $0.3618(58)$ \\
& 20 & 4300 & $0.369(14)$ \\
\hline 3.0 & 4 & 12000 & $0.5427(17)$ \\
& 6 & 51200 & $0.5193(14)$ \\
& 8 & 15500 & $0.5113(44)$ \\
& 12 & 48500 & $0.4958(36)$ \\
& 16 & 19600 & $0.4941(74)$ \\
& 20 & 10400 & $0.486(23)$ \\
\hline 3.5 & 4 & 15000 & $0.6769(16)$ \\
& 6 & 51200 & $0.6495(14)$ \\
& 8 & 17800 & $0.6367(37)$ \\
& 12 & 57000 & $0.6244(34)$ \\
& 16 & 18700 & $0.6105(66)$ \\
& 20 & 4590 & $0.613(23)$ \\
\hline 4.5 & 4 & 15000 & $0.9370(14)$ \\
& 6 & 51200 & $0.9067(14)$ \\
& 8 & 14000 & $0.8884(41)$ \\
& 12 & 63500 & $0.8849(33)$ \\
& 16 & 33741 & $0.8671(52)$ \\
& 20 & 8500 & $0.878(19)$ \\
\hline & 4 & 12000 & $1.8221(23)$ \\
& 6 & 51200 & $1.7904(13)$ \\
& 12 & 14000 & $1.7619(40)$ \\
& 20 & 14100 & $1.7492(41)$ \\
& & & \\
& 12805 & $1.7258(90)$ \\
& & \\
& & \\
& &
\end{tabular}

TABLE II: The measured $1 / g^{2}(L)$ and the number of hybrid Monte Carlo trajectories used, $N_{\text {traj., }}$ at each lattice size $N$ and $\beta_{L}$.

HMC algorithm remains better than $84 \%$ for all cases. For comparison, we made also small-statistics study of the coupling using two flavours of fundamental fermions. These results are shown in Fig. 2 .

What can we conclude from these figures? We remind that we can directly compare physical lattice sizes only at fixed $\beta_{L}$; different $\beta_{L}$ correspond to very different lattice spacings. In the continuum $L$ is the sole physical scale, and $g^{2}(L)$ must be monotonic (or equivalently, $L$ is a function of $g^{2}$ through dimensional transmutation). Thus, the non-monotonic behaviour of data seen at $\beta_{L}=2.05$ and 2.2 is caused by finite lattice spacing 


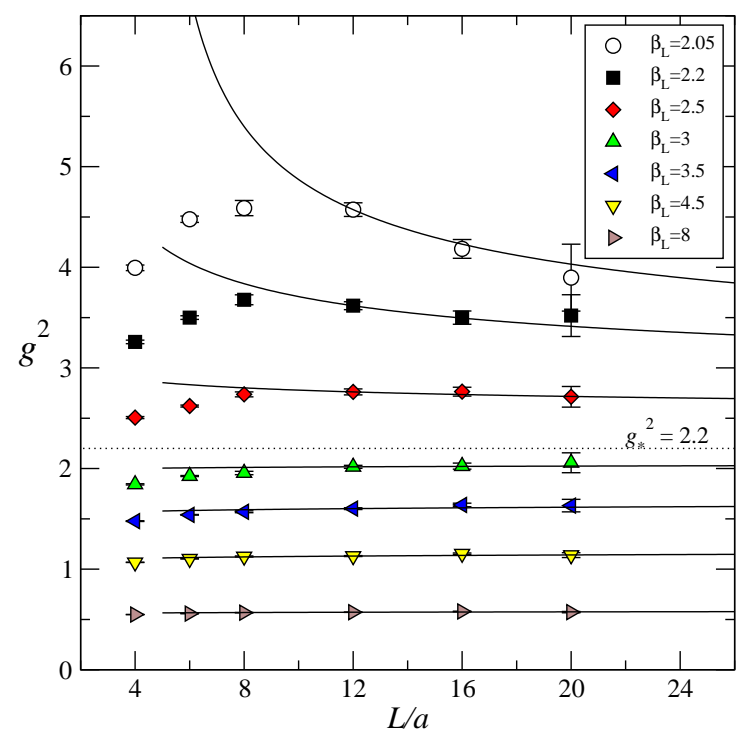

FIG. 1: Lattice measurements of $g^{2}\left(L / a, \beta_{L}\right)$. Continuous lines show $g^{2}(L)$ integrated from Eq. (19), constrained to go through lattice points at $L / a=12$.

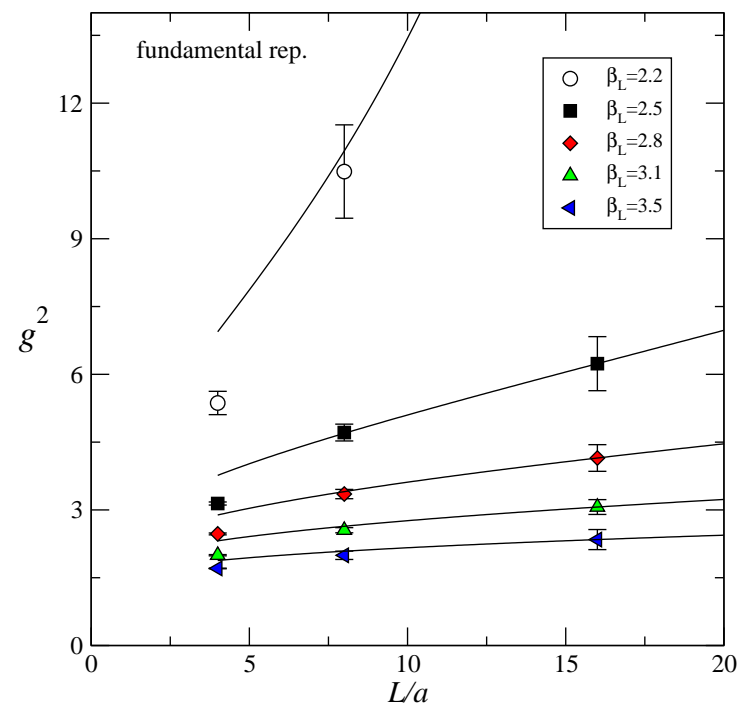

FIG. 2: Small-statistic measurements of $g^{2}\left(L / a, \beta_{L}\right)$ for 2 flavours of fundamental representation fermions, to be contrasted with the adjoint representation case in Fig. 1 The value of $g^{2}$ at $\beta_{L}=2.2, L / a=16$ is $26 \pm 7$. Continuous lines show $g^{2}(L)$ integrated using 2-loop $\beta$-function, constrained to go through lattice points at $L / a=16$.

effects, i.e. too small $L / a$. Hence, we do not include lattices with $L / a \leq 8$ in subsequent analysis.

Our most significant result is readily evident from Fig. 1. $g^{2}\left(L / a, \beta_{L}\right)$ is an increasing function of $L$ at $\beta_{L} \gtrsim 3,\left(g^{2} \lesssim 2.2\right)$, but decreasing at $\beta_{L} \lesssim 2.5\left(g^{2} \gtrsim 2.2\right)$ at large volumes. This is precisely the expected behaviour for a theory with an infrared fixed point and markedly different from the asymptotically free QCDlike behaviour in Fig. 2. In this case $g^{2}(L)$ is always

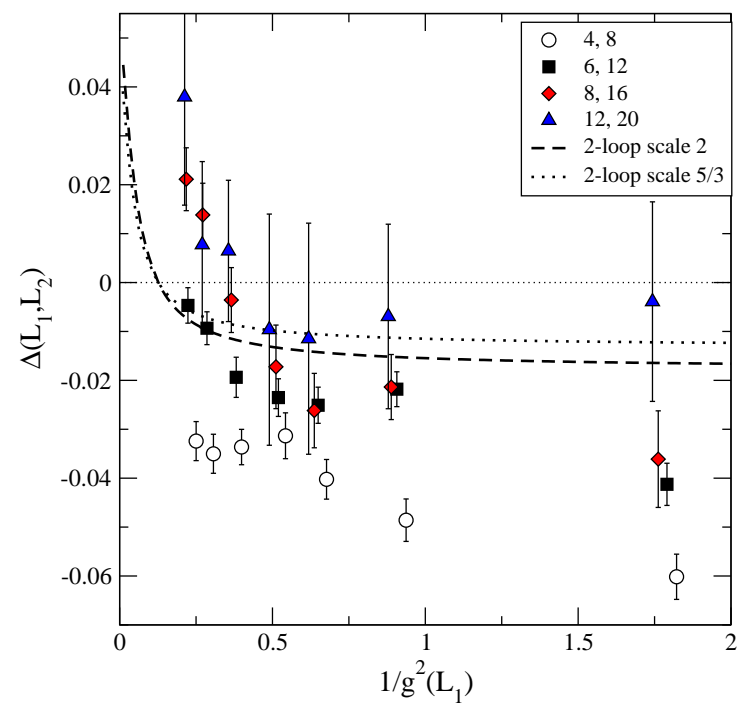

FIG. 3: Step scaling $\Delta\left(L_{1}, L_{2}, \beta_{L}\right)$ for different pairs of volumes, see Eq. (18), plotted against $1 / g^{2}\left(L_{1}, \beta_{L}\right)$. The scaling factor is 2, except for the pair with largest volumes. Also shown is the 2-loop perturbative result for corresponding ratios of volumes.

increasing, and the larger $g^{2}$ is, the steeper the increase. For the adjoint representation the observed fixed point $g_{*}^{2} \sim 2 \ldots 3$ is significantly below the two-loop result 7.9.

Further analysis is needed in order to estimate the full $\beta$-function. The standard method proceeds through the measurement of step scaling functions: using lattices of sizes $L$ and $s L$, where $s$ is a scale factor, we measure $g^{2}\left(L / a, \beta_{L}\right)$ and $g^{2}\left(s L / a, \beta_{L}\right)$, thus obtaining a discrete step of the evolution of $g^{2}(L)$. Now we can search for $\beta_{L}^{\prime}$ so that $g^{2}\left(L / a, \beta_{L}^{\prime}\right)=g^{2}\left(s L / a, \beta_{L}\right)$. Repeating these scaling and matching steps the evolution of $g^{2}$ over a very large range of scales can be covered. The continuum limit extrapolation is enabled by redoing the analysis on different original $L / a$ [33]. This method has been successfully used, e.g. to determine the running coupling in $\mathrm{QCD}$ 24.

In Fig. 3 we show the step scaling using the quantity

$$
\Delta\left(L_{1}, L_{2}, \beta_{L}\right)=\frac{1}{g^{2}\left(L_{2}, \beta_{L}\right)}-\frac{1}{g^{2}\left(L_{1}, \beta_{L}\right)} .
$$

We use scaling factor $s=L_{2} / L_{1}=2$, except for the largest volume $L / a=20$, where we do not have $L / a=10$ data avalable and use $s=5 / 3$. We also show the 2-loop scaling result computed from Eq. (2).

If finite lattice spacing effects were absent, all points with the same $s$ should fall on a universal curve. This is clearly not the case; the small volume data lies significantly below the large volume points. We do not attempt to extrapolate the data to infinite volume (continuum limit), because we do not trust the smallest volume data to be close enough of continuum behaviour. Nevertheless, $\Delta\left(L_{1}, L_{2}, \beta_{L}\right)$ becomes negative at small $1 / g^{2}$, indicating the existence of the infrared fixed point. 
The main reason for the difficulty of using the step scaling is that the evolution of $g^{2}(L)$ is very slow, much slower than with fundamental fermions. The slow evolution is easily masked by finite lattice spacing artifacts at small $L / a$, whereas the faster evolution in fundamental fermion theory is rather easily observable. Improved actions can be expected to help significantly here. The slow evolution also increases the number of required recursive steps in the step scaling method and hence statistical errors.

Due to the numerical problems with the step scaling function mentioned above, we will therefore also consider the following alternative method: we use the measured values of $g^{2}\left(L / a, \beta_{L}\right)$ on volumes $12^{4}-20^{4}$, and fit a $\beta$-function ansatz to the data. The ansatz includes the small- $g^{2}$ perturbative part and it should have only a few tunable parameters. The aim is to find an envelope of continuum $\beta$-functions which are consistent with the measurements. The underlying assumption is that the lattice artifacts are already sufficiently small at $L / a=12$. This should be checked with new simulations with improved actions in the future.

The simplest ansatz is the perturbatively motivated one $\beta(g)=-b_{0} g^{3}-b_{1} g^{5}-b_{2} g^{7}$. Here $b_{0}$ and $b_{1}$ are fixed by the perturbative $\beta$-function Eq. (2), and $b_{2}$ is a fit parameter. This function is actually consistent with the data, but it is very likely too constrained giving unrealistically small error range. In order to have independent parameters for the value of the fixed point coupling and the slope of the $\beta$-function at the fixed point, we are led to the following ansatz:

$$
\beta(g)=-b_{0} g^{3}-b_{1} g^{5}+\left(b_{0} g_{*}^{3-\delta}+b_{1} g_{*}^{5-\delta}\right) g^{\delta} .
$$

Again $b_{0}$ and $b_{1}$ are fixed by the perturbation theory and $g_{*}$ and $\delta$ are fit parameters which determine the fixed point and the slope of the $\beta$-function around the fixed point. The ansatz is naturally not of asymptotic form at small $g^{2}$, but this does not matter for our purposes: the $g^{\delta}$-term turns out to be negligible at small $g^{2}$, and the purpose of the ansatz is to approximate the true $\beta$ function in the vicinity of the fixed point. In practice equivalent results are obtained using perturbatively motivated ansatz $\ldots b_{2} g^{7}+b_{3} g^{9}$; however, Eq. (19) offers more freedom for the slope at $g^{2}=g_{*}^{2}$.

We perform the fit as follows: integrating $\beta(g)=$ $-L \mathrm{~d} g(L) / \mathrm{d} L$ and constraining $g^{2}(L)$ to the lattice measurements at $L / a=12$ for each $\beta_{L}$ gives us the continuous lines shown in Fig. 10. The integrated $g^{2}\left(L / a, \beta_{L}\right)$ are compared with the lattice measurements at $L / a=16$ and 20, giving us a $\chi^{2}$ criterion for the fit. Good fits $\left(\chi^{2} /\right.$ d.o.f $\left.\sim 1\right)$ are obtained when parameters vary from

\footnotetext{
2 Strictly speaking the fit is not optimal due to the constraint at $L / a=12$. Relaxing this constraint gives better $\chi^{2}$, at the cost of a more complicated fit procedure. However, because $L / a=12$ has smaller errors than larger volumes the effect is small.
}

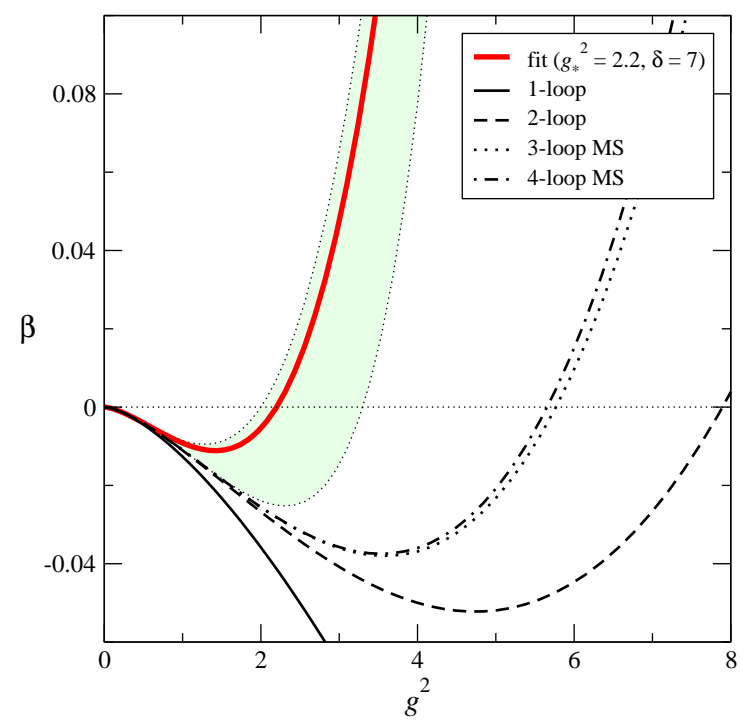

FIG. 4: The $\beta$-function obtained from Eq. (19), with $g_{*}^{2}=2.2$. The shaded area shows the estimated error range of the fit. Shown are also universal perturbative one- and two-loop $\beta$ functions, together with the three- and four-loop results in the MS-scheme [25]. Because of the different scheme, these are not directly comparable with the lattice (SF-scheme) results.

$\left(g_{*}^{2}, \delta\right) \approx(2.0,6)$ to $(3.2,15)$ in a narrow region. Thus, the parameters are strongly correlated. The slope of the $\beta$-function at the fixed point is a universal quantity, but it is only weakly constrained:

$$
\left[g \frac{\mathrm{d} \beta(g)}{\mathrm{d} g}\right]_{g^{2}=g_{*}^{2}}=0.12 \ldots 0.42 .
$$

We take $g_{*}^{2}=2.2, \delta=7$ to be our benchmark value, which is motivated by the fact that $\delta=7$ is the order of the NNLO perturbative $\beta$-function. These lines are shown in Fig. 1) However, we by no means imply that the fitted value gives the perturbative result; it only quantifies all unknown contributions. If we fix $\delta=7, g_{*}^{2}$ can vary between 2.05 and 2.4 .

The result of the fitting procedure is shown in Fig. 4. together with the error band formed by the variation of the parameters in the region where acceptable fit is obtained. While the error band is wide, the qualitative features agree with the corresponding step scaling functions calculated from the measurements; both methods indicate positive $\beta(g)$ at large $g^{2}$. We also note that the two-loop perturbative $\beta$-function alone fits the measurements well at $\beta_{L} \gtrsim 3\left(g^{2} \lesssim 2\right)$.

Fig. 4 also shows the three- and four-loop MS-scheme results [25]. Because of the different scheme, these are not directly comparable with the SF-scheme results obtained in our simulations - for example, the value of $g_{*}^{2}$ is scheme dependent. However, it is interesting that also in the MS-scheme the fixed point is at substantially smaller 


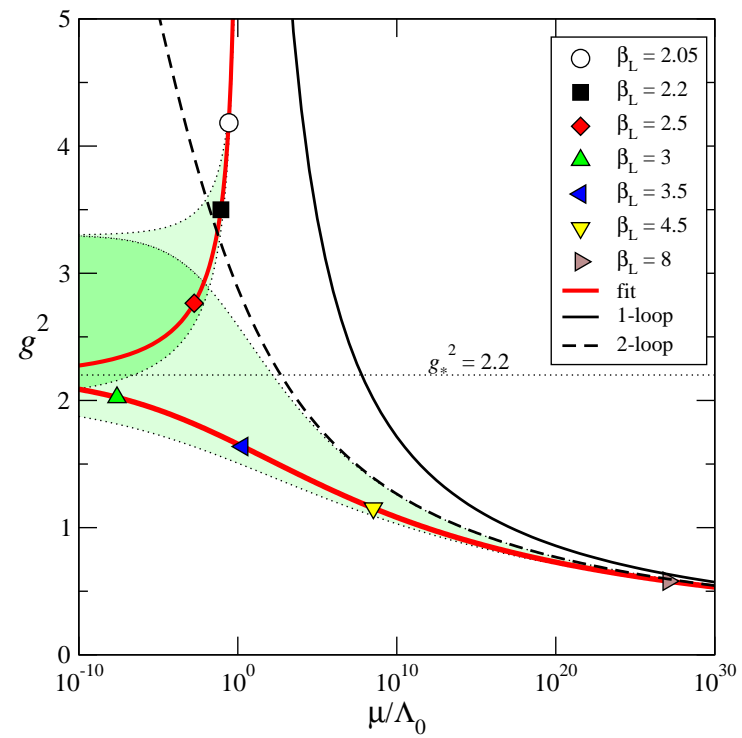

FIG. 5: $g^{2}(\mu=1 / L)$ determined from Eq. (19), together with perturbative $g^{2}$. Scale $\Lambda_{0}$ is determined so that $\mu=\Lambda_{0}$ is at the IR Landau pole of the asymptotically free one-loop coupling and at the UV Landau pole of the measured nonasymptotically free branch $\left(g^{2}>g_{*}^{2}\right)$. For illustration, the $L / a=16$ lattice points have been placed on the benchmark curve at the corresponding $g^{2}$-values, indicating the relative scale hierarchy between simulations.

$g_{*}^{2}$ than indicated by the universal two-loop calculation. ${ }^{3}$

Finally, the fitted $\beta$-function can be integrated to obtain the coupling $g^{2}$. This is shown in Fig. 5 as a function of $\mu=1 / L$. The result is split into two branches: the asymptotically free one with $g^{2}<g_{*}^{2}$, and the not asymptotically free with $g^{2}>g_{*}^{2}$. These branches are disconnected: when the $\beta$-function is integrated to obtain $g^{2}(\mu)$, the integration constants are independent on both sides of the fixed point. Thus, the scaling between the $\mu$-values between the branches is arbitrary in Fig. 5

Finally, we note that the two-loop perturbative $\beta$ function alone gives us an acceptable fit if we ignore the data at $\beta_{L} \leq 2.5\left(g^{2} \gtrsim 2.7\right)$. Indeed, in that case it is possible to fit a $\beta$-function which does not feature an IR fixed point. Thus, the evidence for the fixed point comes from the data at $\beta_{L}=2.05$ and 2.2. These are at relatively strong bare lattice coupling, and one can expect large finite $a$ effects. This can be clearly seen at small $L / a$ in Fig. 1. Nevertheless, as $L / a \rightarrow \infty$ at fixed $\beta_{L}$, the scale hierarchy between the physically interesting scale $L$ and the lattice spacing $a$ increases, and the evolution of the coupling constant approaches the continuum one.

\section{CONCLUSIONS AND OUTLOOK}

Let us now discuss the interpretation of these results. We note that the observed range for the critical coupling, $g_{*}^{2} \sim 2.0-3.2$ is significantly below the perturbative estimate $g_{*}^{2} \sim 8$ from Eq. (2). Similar behaviour has been observed in $\mathrm{SU}(3)$ gauge theory with 2-index symmetric fermions [14]. The obvious question is why the perturbative two-loop $\beta$-function fails although the nonperturbative analysis implies that $\alpha_{*}=g_{*}^{2} / 4 \pi$ is small. Since the perturbative determination of the fixed point is based on the competing effects from one- and two-loop orders, it is reasonable to expect that higher loop orders can contribute with similar magnitude and one needs nonperturbative analysis. This is seen in the MS-scheme results in Fig. 4. Beyond two loops the value of $g_{*}^{2}$ is scheme dependent; our result is in SF scheme. Comparison of the nonperturbative result and the perturbative curves in Fig. 固 demonstrates that even if the actual value of the coupling at the fixed point is small, the nonperturbative effects can be large.

When we combine the results of the analysis here with the spectrum measurements of ref. [26], a consistent picture emerges. In the whole parameter range where simulations with massless fermions are possible the infrared behaviour is controlled by the conformally invariant fixed point $g_{*}^{2}$ and there is no chiral symmetry breaking. If $g^{2}<g_{*}^{2}$, the theory is asymptotically free, otherwise not. The conformal invariance can be explicitly broken by e.g. adding a small fermion mass, which can lead to a "walking" type evolution.

As discussed above, for the lattice action used here it has been observed that it is not possible to take the limit $m_{Q} \rightarrow 0$ when $\beta_{L} \lesssim 2$. Thus, the $\beta_{L}=2.05$ points in Fig. 5 are close to the smallest $\beta_{L}$ where the conformal behaviour can be studied. This limitation is a non-universal lattice artifact, and may differ for some other fermion discretisation. This motivates a study with fully $O(a)$ improved lattice formalism.

Acknowledgement: KR acknowledges the support of Academy of Finland grant 114371. AH acknowledges the support by the NFS under grant number PHY-055375 and DOE grant under contract DE-FG02-01ER41172. The simulations were performed at the Center for Scientific Computing (CSC), Finland, and the total computing effort amounts to $\sim 10^{18}$ flop.
[1] C. T. Hill and E. H. Simmons, Phys. Rept. 381, 235 (2003) [Erratum-ibid. 390, 553 (2004)] arXiv:hep-ph/0203079; F. Sannino, arXiv:0804.0182 [hep-ph].

[2] H. Georgi, Phys. Rev. Lett. 98, 221601 (2007)
arXiv:hep-ph/0703260; H. Georgi, Phys. Lett. B 650, 275 (2007) arXiv:0704.2457 [hep-ph]].

[3] F. Sannino and R. Zwicky, Phys. Rev. D 79, 015016 (2009) arXiv:0810.2686 [hep-ph]].

[4] H. Pagels, Phys. Rept. 16, 219 (1975). 
[5] R. Fukuda and T. Kugo, Nucl. Phys. B 117, 250 (1976).

[6] T. Appelquist, K. D. Lane and U. Mahanta, Phys. Rev. Lett. 61, 1553 (1988).

[7] T. A. Ryttov and F. Sannino, Phys. Rev. D 78, 065001 (2008) arXiv:0711.3745 [hep-th]].

[8] D. D. Dietrich, arXiv:0908.1364 [hep-th].

[9] O. Antipin and K. Tuominen, arXiv:0909.4879 [hep-ph].

[10] E. Poppitz and M. Unsal, JHEP 0909, 050 (2009) arXiv:0906.5156 [hep-th]]; E. Poppitz and M. Unsal, arXiv:0910.1245 [hep-th].

[11] F. Sannino and K. Tuominen, Phys. Rev. D 71, 051901 (2005) arXiv:hep-ph/0405209; D. D. Dietrich, F. Sannino and K. Tuominen, Phys. Rev. D 72, 055001 (2005) arXiv:hep-ph/0505059; D. D. Dietrich and F. Sannino, Phys. Rev. D 75, 085018 (2007) arXiv:hep-ph/0611341.

[12] T. A. Ryttov and F. Sannino, Phys. Rev. D 78, 115010 (2008) arXiv:0809.0713 [hep-ph]].

[13] S. Catterall and F. Sannino, Phys. Rev. D 76, 034504 (2007) arXiv:0705.1664 [hep-lat]]; S. Catterall, J. Giedt, F. Sannino and J. Schneible, JHEP 0811, 009 (2008) arXiv:0807.0792 [hep-lat]].

[14] Y. Shamir, B. Svetitsky and T. DeGrand, Phys. Rev. D 78, 031502 (2008) arXiv:0803.1707 [hep-lat]]; T. DeGrand, Y. Shamir and B. Svetitsky, Phys. Rev. D 79, 034501 (2009) arXiv:0812.1427 [hep-lat]].

[15] L. Del Debbio, B. Lucini, A. Patella, C. Pica and A. Rago, Conformal vs confining scenario in SU(2) with adjoint fermions, arXiv:0907.3896 [hep-lat]; L. Del Debbio, A. Patella and C. Pica, Higher representations on the lattice: numerical simulations. SU(2) with adjoint fermions, arXiv:0805.2058 [hep-lat];

[16] Z. Fodor, K. Holland, J. Kuti, D. Nogradi and C. Schroeder, Chiral properties of $S U(3)$ sextet fermions, arXiv:0908.2466 [hep-lat].

[17] F. Bursa, L. Del Debbio, L. Keegan, C. Pica and T. Pickup, Running of the coupling and quark mass in $S U$ (2) with two adjoint fermions, arXiv:0910.2562 [hep- $\mathrm{ph}]$.

[18] D. K. Sinclair and J. B. Kogut, arXiv:0909.2019 [hep-lat].

[19] T. Appelquist, G. T. Fleming and E. T. Neil, Phys. Rev. Lett. 100, 171607 (2008) arXiv:0712.0609 [hepph]]; T. Appelquist, G. T. Fleming and E. T. Neil, Phys. Rev. D 79, 076010 (2009) arXiv:0901.3766 [hep-ph]].

[20] Z. Fodor, K. Holland, J. Kuti, D. Nogradi and C. Schroeder, arXiv:0907.4562 [hep-lat]

[21] A. Deuzeman, M. P. Lombardo and E. Pallante, arXiv:0904.4662 [hep-ph].

[22] X. Y. Jin and R. D. Mawhinney, arXiv:0910.3216 [heplat].

[23] A. Hasenfratz, Phys. Rev. D 80, 034505 (2009) arXiv:0907.0919 [hep-lat]].

[24] M. Della Morte, R. Frezzotti, J. Heitger, J. Rolf, R. Sommer and U. Wolff [ALPHA Collaboration], Nucl. Phys. B 713, 378 (2005) arXiv:hep-lat/0411025.

[25] T. van Ritbergen, J. A. M. Vermaseren and S. A. Larin, Phys. Lett. B 400, 379 (1997) arXiv:hep-ph/9701390.

[26] A. J. Hietanen, J. Rantaharju, K. Rummukainen and K. Tuominen, JHEP 0905, 025 (2009) arXiv:0812.1467 [hep-lat]].

[27] A. Hietanen, J. Rantaharju, K. Rummukainen and K. Tuominen, PoS LATTICE2008, 065 (2008) arXiv:0810.3722 [hep-lat]].

[28] F. Sannino, arXiv:0811.0616 [hep-ph].

[29] M. Luscher, S. Sint, R. Sommer, P. Weisz and U. Wolff, Nucl. Phys. B 491, 323 (1997) arXiv:hep-lat/9609035.

[30] M. Luscher, P. Weisz and U. Wolff, Nucl. Phys. B 359, 221 (1991).

[31] M. Luscher, R. Narayanan, P. Weisz and U. Wolff, Nucl. Phys. B 384, 168 (1992) arXiv:hep-lat/9207009

[32] S. Sint, Nucl. Phys. B 451, 416 (1995) arXiv:hep-lat/9504005.

[33] M. Luscher, R. Sommer, U. Wolff and P. Weisz, Nucl. Phys. B 389, 247 (1993) arXiv:hep-lat/9207010. 